\title{
Analysis on correlation between the Earth's Rotation and Earthquakes Preceding the Occurrence of the 2019 M7.1 Ridgecrest Earthquake
}

\author{
Yane Li \\ Institute of Geophysics,CEA,China \\ Xuezhong Chen ( $\nabla$ cxz8675@163.com ) \\ Institute of geophyscis,CEA,China https://orcid.org/0000-0003-2228-6567
}

Full paper

Keywords: The M7.1 Ridgecrest earthquake, Earth's rotation, correlation, Schuster test,two-rate Poisson model

Posted Date: June 14th, 2020

DOI: https://doi.org/10.21203/rs.3.rs-30872/v2

License: (c) (1) This work is licensed under a Creative Commons Attribution 4.0 International License.

Read Full License 
2 Analysis on correlation between the Earth's Rotation and Earthquakes

$3 \quad$ Preceding the Occurrence of the 2019 M7.1 Ridgecrest Earthquake

4

5

6

7

8

Yane Li, Xuezhong Chen*

\section{Abstract}

For earthquakes $(M \geq 2.0)$ occurring in and around the rupture zone of the $M 7.1$ Ridgecrest earthquake between January 1990 and June 2019, we analyzed the correlation between the phase of Earth's rotation and the occurrence of earthquakes. The Schuster's test and the two-rate Poisson model were used to statistically examined the correlation between the phase of Earth's rotation and the occurrence of earthquakes. For the Schuster's test,the results were judged based on the $P$-value. When the $P$-value is smaller, the correlation is higher. In general, if $P \leq 5 \%$,the correlation is significant. For the two-rate Poisson model,the results were evaluated by $R$. When the $95 \%$ confidence lower limit on $R\left(R_{L}\right)$ is over one, earthquakes could be non-random.

We calculated the temporal variation on the $P$-value for earthquakes that occurred in the study region and found some lower $P$-values,corresponding significant correlation, for earthquakes $(2.0 \leq M \leq 2.4)$ occurring before the $M 7.1$ event. When the $P$-value reached to the lowest,earthquakes are prone to occur when the Earth's acceleration of rotation is increasing or near the peak acceleration.This correlation is also confirmed by the two-rate Poisson model.

The spacial distribution of the $P$-values for earthquakes $(2.0 \leq M \leq 2.4)$ occurring from Jul. 2013 to Jun. 2019 shows that areas of low $P$-value were located mainly in and around the

\footnotetext{
*Corresponding author, cxz8675@163.com Institute of Geophysics, CEA, No.5 Minzudaxue Nanlu, Haidian District, Beijing 100081, P. R. China
} 
northwest segment of the rupture zone of the M7.1 Ridgecrest earthquake.

The lower $P$-value obtained in this study uncovers the Earth's rotation-triggered earthquakes in and around the rupture zone prior to the M7.1 Ridgecrest earthquake, is possible to be considered as one of its precursors.

Key words:The M7.1 Ridgecrest earthquake, Earth's rotation, correlation, Schuster test,two-rate Poisson model

\section{Introduction}

When the tectonic stress in the Earth's crust increases to a critical value to liberate a stronger earthquake, the focal fault could become extremely unstable where the stronger earthquake would be triggered by a change of stress. Cyclic stress variations in the crust are caused by the Earth's tide and rotation. Although these stress variations are small in comparison to tectonic stress, their rates are often larger than the rates of accumulation for tectonic stress. With regards to tidal stress considered as one of the possible triggers for earthquakes, many scientific studies have been conducted to investigate the correlation between stronger earthquake occurrence and the Earth's tide. Some researches had found that tidal stress has significant effect on triggering earthquakes (e.g., Heaton 1975; Gao et al. 1981; Tsuruoka et al. 1995; Wu et al. 1999; Tanaka et al. 2002a; Cochran et al. 2004; Zhang et al. 2007; Li et al. 2011; Xu et al. 2011; Sun et al. 2014; Bucholc et al. 2016), but some researches had revealed no significant correlation between tidal stress and earthquake occurrence (e.g., Simpson 1957; Knopoff 1964; Shlien et al. 1970; Shudde et al. 1977; Heaton 1982; Stothers 1990; Rydelek 1992; Emter 1997; Vidale et al. 1998),therefore,the tidal triggering of earthquakes still remains controversial. But some researches showed that highly significant tidal triggering of small earthquakes or foreshocks may be expected to appear more clearly prior to a stronger earthquake (Xu et al. 1981; Tanaka et al. 2002b; Tanaka 2010, 2012). Relationship between the occurrence of earthquakes and the Earth's rate of rotation have been investigated since the late 1960s. It has been found that there is a significant correlation between the irregular variation of the rate at which the Earth rotates and seismic energy released by strong earthquakes of medium and deep sources(Stoyko et al. 1969). Seismic activity in the globe is 
triggered by the increase in the Chandler wobble amplitude, which accelerates the movement of the plate to eventually result in great decoupling earthquakes (Kanamori 1977). A positive correlation was revealed between monthly incidence of earthquakes $\left(M_{\mathrm{s}} \geq 7.0\right)$ and the seasonal variations in the Earth's rotation rate(Gao 1981), whereas earthquakes $\left(M_{\mathrm{s}} \geq 7.5\right)$ that occur on an annual basis have a negative correlation with the 10-year variations in the Earth's rate of rotation (Ma et al. 2007). Earthquakes in different regions could have different relations with the variations in the Earth's rate of rotation (e.g., Li et al. 1973; Yu et al. 1974; Fu 1984; Zhao 1990; Zheng et al. 1995; Fu et al. 2004).

The seasonal changes of length of day are mainly caused by atmospheric effects (Richard et al. 1985; Zharov 1995; Höpfner 1998). The amplitude of seasonal change of length of day is approximately $1 \mathrm{~ms}$, the corresponding relative change for angular velocity of Earth's rotation is about $1 \times 10^{-8}$. The induced stress by this change value is roughly $0.2 \mathrm{~Pa}$ on the focal fault plane of the 2019 M7.1 Ridgecrest event, calculated by means of method proposed by Chen et al. in 2018 (Chen et al. 2018). It's quite apparent that the induced stress is too weak to trigger earthquakes. The amplitude of $300 \mathrm{~Pa}$ for stress induced by hydrosphere loading is still negligible compared with the stress drop of strong earthquakes(Lowry 2006). It was found based on slider-block models of slip that slip can amplify dramatically under the action of given stress within a narrow band of resonant periods (Perfettini et al. 2001). Slip response amplifies significantly for a narrow range of periods near the critical period $T_{\mathrm{c}}$ when stiffness $k$ is equal to the critical stiffness $k_{\mathrm{c}}$, and at shorter periods when $k$ is greater than $k_{\mathrm{c}}$ (Lowry 2006). Therefore, for convenience, we can use the seasonal change of the Earth's rotation rate to analyze triggering of earthquakes to avoid the complicated calculation of the stress induced by meteorological processes. We have investigated the correlation between the occurrence of small earthquakes occurring in and around the rupture zones of the Tangshan $M_{s} 7.8$, the Wenchuan $M_{s} 8.0$ and the $2011 M_{w} 9.1$ Tohoku-Oki Japan mainshocks in pre-quake and the phase of Earth's rotation (Wei et al. 2018; Chen et al. 2019,2020). In this study, we will focus on the M7.1 Ridgecrest earthquake in eastern California,USA (date of occurrence: July 6, 2019) to examine the correlation between the changes of the Earth's rotation rate and the occurrence of earthquakes occurring in the Ridgecrest rupture zone and its surrounding region 
prior to the occurrence of the M7.1 Ridgecrest earthquake.

\section{Data and the Study Region}

On July 6th, 2019, the M7.1 Ridgecrest earthquake occurred in southwest of Searles Valley, eastern California. Its focal mechanism solutions indicate a steeply dipping fault plane striking NW-SE with a right lateral slip, or one striking SW-NE with left lateral slip . Aftershocks of the Ridgecrest mainshock spread along the direction of NW-SE(Fig. 1a). According to this, the Ridgecrest earthquake ruptured on a right-slip plane striking NW-SE, due to the relative motion between the Pacific plate and the north American plate. Fig.1b shows the spacial number distribution for the aftershocks in Fig. 1a. It looks like a dumbbell in the middle of which the M7.1 Ridgecrest event is located.

We use earthquakes in the USGS earthquake catalog (https://earthquake.usgs.gov/earthquakes/search/). In analysis,we considered an area of 150 $\mathrm{km} \times 60 \mathrm{~km}$ containing the rupture of the $M 7.1$ Ridgecrest earthquake as the study region, we selected earthquakes of $M \geq 2.0$ that occurred within the study region from January 1990 to Jun 2019.The study region is shown by dashed lines in Fig.2,while earthquakes $(M \geq 2.0)$ that occurred from January 2000 to Jun 2019 were also plotted in Fig.2. On the basis of the G-R relation it can be considered that earthquakes of $M \geq 2.0$ in the study region should be complete since 1990 (Fig. 3). Using this catalog with aftershocks removed, we can assess the changes in the influence of Earth's rotation over the past three decades.In total, 2704 earthquakes were analyzed herein (Fig. 4).

The data of length of day relating to the Earth's rotation was acquired from the Earth Orientation Center(EOC) [http://hpiers.obspm.fr/eop-pc/]. The raw data of length of day with the standard length of day (24 hours) subtracted is shown in Fig. 5a. The change of length of day consist of different periodic components, mainly include the long period component (period over ten years), the seasonal component (period over one month and not more than one year) and the short period component (period not more than one month). We will use the seasonal component to investigate the correlation between the Earth's rotation and the occurrence of earthquakes occurring prior to the M7.1 Ridgecrest event in this paper. The 
seasonal change in the length of day is plotted in Fig. 5b, obtained by band-pass filtering with period band between $120 \mathrm{~d}$ and $370 \mathrm{~d}$.

\section{Analytical Method}

The Schuster's test and the two-rate Poisson model were used in this study to statistically examined the correlation between the phase of Earth's rotation and the occurrence of earthquakes.The former has been widely applied to investigate tidal triggering of earthquakes (Tanaka et al. 2002b; Tanaka 2010, 2012). From a temporal change series of length of day, the phase angle of Earth's rotation at the occurring time of each earthquake can be calculated. The phase angle at each maximum of the length of day is defined as $0^{\circ}$, at the first minimum on the left of the maximum as $-180^{\circ}$, and at the first minimum on the right one as $180^{\circ}$. The phase angle $\theta$ at the occurring time of an earthquake was calculated according to the following formula.

$$
\left.\begin{array}{rlrl}
\theta & =-180^{\circ} \times \frac{t_{0}-t_{e}}{t_{0}-t_{-180}}, & & t_{e}<t_{0} \\
& =180^{\circ} \times \frac{t_{e}-t_{0}}{t_{180}-t_{0}}, & & t_{e} \geq t_{0}
\end{array}\right\}
$$

Where $t_{\mathrm{e}}$ is the occurring time of an earthquake, and $t_{0}$ the time at the maximum of length of day immediately before or after $t_{\mathrm{e}} \cdot t_{-180}$ and $t_{180}$ are the times at the minimum of length of day immediately before and after $t_{\mathrm{e}}$ respectively (Fig. 6). With the phase angles of all earthquakes, we can statistically judge whether there is a significant correlation between those earthquakes and the Earth's rotation not only by use of the Schuster's test (Tsuruoka et al. 1995; Heaton 1975) , but also by use of the two-rate Poisson model (Vidale et al. 1998; Gu et al. 2008; Bucholc et al. 2016).

For the Schuster's test the results are valued by $P$-value between 0 and 1 . In general, if $P \leq$ $5 \%$, the earthquakes could be non-random. The $P$-value of $N$ earthquakes can be obtained on the base of the following formulas. 


$$
\left.\begin{array}{l}
P=e^{-\frac{r^{2}}{N}} \\
r=\sqrt{\left(\sum_{i=1}^{N} \sin \theta_{i}\right)^{2}+\left(\sum_{i=1}^{N} \cos \theta_{i}\right)^{2}}
\end{array}\right\}
$$

Where, $\theta_{1}$ is the phase angle of the i-th earthquake. $N>10$. During the period between point $\mathrm{A}$ and point $\mathrm{B}\left(-180^{\circ} \leq \theta \leq 0^{\circ}\right)$ in Fig. 6, the length of day ascends, the Earth's rotation decelerates, and the length of day descends from point $\mathrm{B}$ to point $\mathrm{C}\left(0^{\circ} \leq \theta \leq 180^{\circ}\right)$, the Earth's rotation accelerates.

For the two-rate Poisson model, the results are valued by $R$.

$$
\left.\begin{array}{l}
R=\frac{N^{+}\left(1-f^{+}\right)}{f^{+}\left(1-N^{+}\right)} \\
R_{L}=e^{\ln R-1.96 A} \\
R_{U}=e^{\ln R+1.96 A} \\
A=\sqrt{\frac{1}{N^{+}}+\frac{1}{N-N^{+}}}
\end{array}\right\}
$$

Where $\mathrm{N}^{+}$is the number of earthquakes which occur within a phase angle range ( denoted by $\theta^{+}$) from $\theta_{a}$ to $\theta_{b} . f^{+}=\frac{\theta^{+}}{360}, \mathrm{~N}$ is the total number of events. The $95 \%$ confidence limits on $R$ are given by $R_{L}$ and $R_{U}$. Generally, if $R_{L}>1, R$ is considered to be significantly greater than one, and the correlation between those earthquakes and the phase angle range $\theta^{+}$is significant.

\section{Results}

From a temporal change series of length of day, we determined the phase angle of Earth's rotation at the occurring time of each earthquake on the base of formula (1), then calculated P-value with formula (2).

We examined the correlation between the seasonal variation of Earth's rotation and the earthquake's occurring. For earthquakes of $2.0 \leq M \leq 2.4$ that occurred in the study region, $P$-value as a function of time can be calculated via considering a six-year time window 
moving by 6 months. A shorter time window maybe introduce random fluctuations, and a longer one maybe smooth the signals that triggered earthquakes.By trial calculation we determined a six-year time window which includes 12 cycles of the seasonal variation in Earth's rotation.We calculated the $P$-value of each window with formula (2), and took the occurring time of the last earthquake in each window as the time of $P$-value. The minimum number of earthquakes in those time windows is 188 , the mean and the maximum are 368 and 657 respectively. Therefore the requirement of earthquake number greater than 10 for the Schuster's test can be better met. Fig. 7a shows $P$-value as a function of time, only four lower $P$-values (less than 1\%) are found within two years prior to the occurrence of the $M 7.1$ Ridgecrest earthquake since 1990. $P$-value dropped rapidly to the lowest value $(0.06 \%)$ when the M7.1 Ridgecrest earthquake was impending.

Each simulated data set and the original experimental one have the same number of events. For each simulated data set the concerned parameters are calculated and then the 95\% confidence ranges of these parameters are also obtained easily. Analysis of the $95 \%$ confidence range of $P$-value for each time window in Fig. 7a was carried out according to bootstrap method with 2000 simulated data sets. The $95 \%$ confidence limit of $P$-value is given by the red area in Fig. 7a, of which the lowest $P$-value was between $0.7 \%$ and $1.0 \%$. Therefore, in the pre-event period, a significant correlation between the occurrence of earthquakes of $2.0 \leq M \leq 2.4$ and the Earth's rotation with confidence level greater than $99 \%$ can be found.

For two hundred and five earthquakes that occurred when $P$-value reached to the lowest, the distribution of phase angle is shown in Fig. $7 \mathrm{~b}$. We found that about $65.37 \%$ of these earthquakes took place when the phase angle was between $-50^{\circ}$ and $130^{\circ}$,corresponding to the later stage of the Earth's deceleration of rotation and the early and middle stages of its acceleration.

$P$-value for earthquakes of $M \geq 2.5$ is also computed as a function of time, the chart is shown in Fig. 8. Six lower $P$-values near $1 \%$ can be found from the figure with the lowest of $0.6 \%$. The $95 \%$ confidence range of $P$-value as resulting from bootstrap analysis is shown by the red area with the lowest of approximately $2.8 \%$.Therefore we can not find any significant correlation between these earthquakes and the Earth's rotation before the M7.1 Ridgecrest 
earthquake, and the six lower $P$-values near 1\% were also not significant.

The spacial distribution of the $P$-values for earthquakes $(2.0 \leq M \leq 2.4)$ occurring from Jul. 2013 to Jun. 2019 is shown in Fig. 9a. A spatial window of $50 \mathrm{~km} \times 50 \mathrm{~km}$ moving by $2 \mathrm{~km}$ in along-latitude and along-longitude directions was considered. When the number of earthquakes in a window is over $15, P$-value can be worked out. Fig. $9 \mathrm{~b}$ shows the spacial distribution of $P<0.5 \%$. It can be found from Fig. $9 \mathrm{~b}$ that low $P$-values located mainly in and around the northwest segment of the rupture zone (marked by the red solid-line frame) of the M7.1 Ridgecrest earthquake.

It is found from Fig. $7 \mathrm{~b}$ that most of earthquakes of $2.0 \leq M \leq 2.4$ took place when the phase angle was between $-50^{\circ}$ and $130^{\circ}$. In the following we used the two-rate Poisson model to test this correlation. We calculated $R$-value with formula (3).For earthquakes of $2.0 \leq M \leq 2.4$ that occurred in the study region, $R$-value as a function of time was calculated via taking a six-year time window moving by 6 months. The result is shown in Fig.10.It can be found from the figure that $R$-value has increased relatively rapidly since the beginning of 2017, and reached the highest value just before the $M 7.1$ Ridgecrest earthquake.The highest $R$ is 1.9, and its corresponding $R_{\mathrm{L}}$ is 1.4. Therefore the correlation between earthquakes of $2.0 \leq M \leq 2.4$ and the phase angles in the range from $-50^{\circ}$ to $130^{\circ}$ is significant before the $M 7.1$ Ridgecrest earthquake.

\section{Discussion and Conclusion}

We analyzed the correlation between the Earth's rotation and earthquakes $(M \geq 2.0)$ occurring prior to the M7.1 Ridgecrest earthquake in and around the rupture zone. For earthquakes of $2.0 \leq M \leq 2.4$ the significant correlation was found mainly in and around the northwest segment of the rupture zone prior to the M7.1 Ridgecrest earthquake. When the $P$-value reached to the lowest in Fig.7b,earthquakes were easier to happen when the phase angle was between $-50^{\circ}$ and $130^{\circ}$. This correlation was also confirmed by the two-rate Poisson model. Figure 11 shows the seasonal variations of LOD (length of day) and $a_{e}$ which is the linear acceleration at the equator. The phase angle range between $-50^{\circ}$ and $130^{\circ}$ is marked by two boxes.It can be seen that this phase angle range corresponds to the increasing stage or near peak value of the 
Earth's acceleration of rotation.

Furthermore we calculated the spacial distribution of the $P$-values for earthquakes $(2.0 \leq M \leq 2.4)$ occurring from Jul. 2013 to Jun. 2019 and found that areas of low $P$-value $(P<0.5 \%)$ were located mainly in and around the northwest segment of the rupture zone of the M7.1 Ridgecrest earthquake.

It is no doubt that not only the variation in the Earth's rotation rate but also the meteorological processes can cause variational stress in the crust. It is a well founded viewpoint that the elastic stiffness of seismogenic faults will change with time.If the elastic stiffness of a fault is equal to the critical elastic stiffness, fault slip response to the action of the changing stress with period near the critical period will amplify significantly (Perfettini etal. 2001; Lowry 2006). In this situation, a strong earthquake could be coming to occur, and part of its focal fault will get exceedingly unstable where most of smaller earthquakes would be in phase with the perturbatioanal stress. But the initial rupture may not have been in this part of the focal fault when the mainshock occurred. Therefore, we would have a conclusion that the lower $P$-value found in this study uncovers that the Ridgecrest focal fault most probably was prone to occurring a strong earthquake, is possible to be considered as one of its precursors .

\section{Acknowledgments}

We used the seismic catalogue data from USGS, the focal mechanism solution from the Global Centroid Moment Tensor Project database, and the data of length of day from the Earth Orientation Center. We express sincerely thanks to the journal editors and anonymous reviewers for their help and beneficial comments to the manuscript.

\section{Authors'contributions}

Both authors carried out the research. X Chen conducted the analyses and prepared the manuscript. Y Li contributed to the data selection of the data, performed data processing, provided interpretation of the results obtained in this study and helped to prepare the manuscript. Both authors read and approved the final manuscript. 


\section{Competing interests}

237 The authors declare that they have no competing interests.

\section{Availability of data and materials}

239

240

Please contact author for data requests.

\section{Funding}

This study was supported by China National Key Research and Development Program (2018YFC1503400).

\section{Ethics approval and consent to participate}

$$
\text { Not applicable. }
$$

\section{Consent for publication}

Not applicable.

\section{References}

Beeler N M, Lockner D A (2003) Why earthquakes correlate weakly with Earth tides: The effects of periodic stress on the rate and probability of earthquake occurrence. J Geophys Res 108: 239. doi:10.1029/2001JB001518

Bucholc M, Steacy S (2016) Tidal stress triggering of earthquakes in Southern California. Geophysical Journal International 205: 681-693

Chen X, Wang H, Wang S, Wei Y, Guo X, Chen L, Li Y (2018) Effect of the Earth's rotation deceleration on the occurrence of the 2008 Wenchuan Ms8.0 earthquake. Earthquake 38: 127-136(in Chinese)

Chen X, Li Y (2019) Relationship Between the deceleration of Earth's rotation and earthquakes that occurred before the Ms 8.0 Wenchuan earthquake. Pure Appl Geophys 
176: 5253-5260. doi:10.1007/s00024-019-02273-6

Chen X, Li Y, Wei Y, Guo X, Chen L (2020) Relationship between the Earth's rotation rate and earthquakes occurring prior to the 2011 Mw9.1 Tohoku-Oki Japan earthquake. Chinese J Geophys 63:440-444. doi:10.6038/cjg2020M0565(in Chinese)

Cochran E S, Vidale J E, Tanaka S (2004) Earth tides can trigger shallow thrust fault earthquakes. Science 306: 1164-1166

Efron B (1979) Bootstrap methods: another look at the jackknife. Ann. Stat 7: 1-26

Emter D (1997) Tidal triggering of earthquakes and volcanic events. In: Wilhelm H, Ztirn W, Wenzel H-G (eds) Tidal Phenomena, Lect Notes Earth Sci 66, Springer-Verlag, Berlin

Fu Z (1984) Distribution of the shallow earthquakes in the island arc-trench zones and the Earth's rotation. Chinese Journal of Geophysics 27: 53-63(in Chinese)

Fu Z, Shao H, Ding X (2004) Relation between distribution of shallow strong earthquake in the Chinese continent and the Earth's rotation rate variation. Earthquake 24: 15-20(in Chinese)

Gao J (1981) Discussion on earthquake triggering of change of the Earth's rotation angular velocity . Chinese Science Bulletin 26: 293-296(in Chinese)

Gao X, Yin Z, Wang W, Huang L, Li J (1981) Triggering of earthquakes by the tidal stress tensor. Acta Seismol Sin 3: 264-275(in Chinese)

Gu K, Ng H K T, Tang M L, Schucany W R (2008) Testing the Ratio of Two Poisson Rates. Biome J 50: 2831-298

Heaton T H (1975) Tidal triggering of earthquakes. Geophys J R Astr Soc 43: 307-326

Heaton T H (1982) Tidal triggering of earthquakes. Bull Seism Soc Am 72: 2181- 2200

Höpfner J (1998) Seasonal variations in length of day and atmospheric angular momentum. Geophys J Int 135: 407-437

Kanamori H (1977) The energy release in great earthquakes. J Geophys Res 82: 2981-2987 
Knopoff J (1964) Earth tides as a triggering mechanism for earthquakes. Bull Seism Soc Am 54: $1865-1870$

Ma L, Han Y, Yin Z (2007) Distribution characteristics of global significant earthquakes and possible connection between earthquakes and Earth's variable rotation rate. Astronomical Research \& Technology 4: 406-411(in Chinese)

Magda B, Sandy S (2016) Tidal stress triggering of earthquakes in Southern California. Geophys J Int 205: 681 - 693

Matsuyama T (2018) An application of bootstrap method for analysis of particle size distribution. Advanced Powder Technology. doi:org/10.1016/j.apt.2018.03.002

Li Q, Xiao X, Li Z (1973) A preliminary study on the relation between the great earthquakes of China and the secular variation of the angular velocity of rotation of the earth. Chinese Journal of Geophysics 16: 71-80(in Chinese)

Li Z, Zhang X (2011) Relationship of tidal stress and large earthquakes. Earthquake 31: 48-57(in Chinese)

Lowry A R (2006) Resonant slow fault slip in subduction zones forced by climatic load stress. Nature 442: 802-805

Ma L, Han Y,Yin Z (2007) Distribution characteristics of global significant earthquakes and possible connection between earthquakes and Earth's variable rotation rate . Astronomical Research \& Technology 4: 406-411(in Chinese)

Perfettini H, Schmittbuhl J, Rice J R, Cocco M (2001) Frictional response induced by time-dependent fluctuations of the normal loading. J Geophys Res 106: 13455-13472

Richard D R, David A S (1985) Contribution of Stratospheric Winds to Annual and Semiannual Fluctuations in Atmospheric Angular Momentum and the Length of Day. J Geophys Res 90: 8033-8041

Rydelek P A, Sacks I S, Scarpa R (1992) On tidal triggering of earthquakes at Campi Flegrei, Italy. Geophys J Int 109: 125-137 
Shlien S, Toksoz M N (1970) A clustering model for earthquake occurrences. Bull Seism Soc Am 60: 1765-1787

Shudde R H, Barr D R (1977) An analysis of earthquake frequency data. Bull Seism Soc Am 67: 1379-1386

Simpson J F (1957) Earth tides as a triggering mechanism for earthquakes. Earth and Planetary Sci Letters 2: 473-478

Stothers R B (1990) A search for long-term periodicities in large earthquakes of Southern and Central California. Geophys Re. Lett 17: 1981-1984

Stoyko A, Stoyko N (1969) Rotation de la terre, phénomènes géophysiques et activité du soleil. Bulletin Academic Royal Belique 5: 279-285

Sun C, Yan C, Wu X, Xu Y (2014) The effect of tidal triggering on seismic fault in eastern Tibetan plateau and its neighboring areas. Chinese J Geophys 57: 2054-2064(in Chinese)

Tanaka S, Ohtake M, Sato H (2002a) Evidence for tidal triggering of earthquakes as revealed from statistical analysis of global data. J Geophys Res 107: ESE 1-1-ESE 1-11

Tanaka S, Ohtake M, Sato H (2002b) Spatio-temporal variation of the tidal triggering effect on earthquake occurrence associated with the 1982 South Tonga earthquake of Mw 7.5. Geophys Res Lett 29. doi:10.1029/2002GL015386

Tanaka S (2010) Tidal triggering of earthquakes precursory to the recent Sumatra megathrust earthquakes of 26 December 2004 (Mw 9.0), 28 March 2005 (Mw 8.6), and 12 September 2007 (Mw 8.5). Geophys Res Lett 37: L02301. doi:10.1029/2009GL041581

Tanaka S (2012) Tidal triggering of earthquakes prior to the 2011 Tohoku-Oki earthquake (Mw 9.1). Geophys Res Lett 39: L00G26. doi:10.1029/2012GL051179

Tsuruoka H, Ohtake M, Sato H (1995) Statistical test of the tidal triggering of earthquakes: Contribution of the ocean tide loading effect. Geophys J Int 122: 183-194

Vidale J E, Agnew D C, Johnston M J S, Oppenheimer D H (1998) Absence of earthquake correlation with Earth tides:An indication of high preseismic fault stress rate. J Geophys 
Wei Y, Chen X, Li Y (2018) Relation between Earth's rotation and small earthquakes occurring before the Ms7.8 Tangshan mainshock. Chinese Science Bulletin 63: 1822-1828(in Chinese)

Wu X, Mao W, Huang Y, Jiang J (1999) Statistical analysis of tidal stress effect on seismic faults. Chinese J Geophys 42: 65-74(in Chinese)

Xu S, Wang B, Jones M L, Ma X, Shen P (1981) The foreshock sequence of Haicheng earthquake and earthquake swarm. Acta Seismol Sin 3: 1-10(in Chinese)

Xu Y, Wu X, Yan C, Huang Y, Wang Y, Li T (2011) The features of tidal Coulomb failure stresses on various kinds of seismic fault. Chinese J Geophys 54: 756-763(in Chinese)

Yu Z, Chen Y, Luo S, Liang S, Xu S, Ye S (1974) Analysis of the relationship between the seasonal variation of earth rotation rate and earthquakes. Chinese J Geophys 17: 44-49(in Chinese)

Zhang J, Qi W, Yang L, Chen R, Wang W (2007) A study on tidal force stress triggering of strong earthquakes. Chinese J Geophys 50: 448-454(in Chinese)

Zhao M (1990) Relationship between the Earth's rotation rate variation and the earthquake rhythm in Mainland China. Chinese Science Bulletin 35: 1402-1405(in Chinese)

Zharov V E (1995) Connection of the Earth's rotation with the atmospheric angular momentum and the strongest earthquakes. Astronomical and Astrophysical Transactions 9: $317-327$

Zheng D, Zhou Y (1995) Study of relationship between change of earth rotation rate and globe earthquakes. Acta Seismol Sin 17: 25-30(in Chinese) 


\section{Captions}

Fig. 1 Maps showing the locations of aftershocks and their spacial number distribution,regional faults are shown as red lines, the red star shows the epicenter of the M 7.1 Ridgecrest earthquake,the focal mechanism solution comes from the Global Centroid Moment Tensor Project [www.globalcmt.org/CMTsearch.html]. (a) The locations of aftershocks $(M \geq 2.0)$ that occurred from 6 July to 31 August following the $M 7.1$ Ridgecrest earthquake. (b) The spacial number distribution for earthquakes in Fig.1a, a spatial window of $0.1^{\circ} \times 0.1^{\circ}$ is moved by $0.01^{\circ}$ in both the along-latitude and along-longitude directions.

Fig. 2 Map showing location of the study region ( surrounded by dashed lines) and its tectonic setting.Regional faults are shown as red lines. The bold black line located at the lower left shows the plate boundary.Black circles show the epicenters of earthquakes $\left(M_{\mathrm{L}} \geq 2.0\right)$ that occurred from January 2000 to Jun 2019. The star represents the epicenter of the $M 7.1$ Ridgecrest mainshock and solid black lines show the rupture zone.

Fig. 3 Cumulative number versus magnitude for earthquakes in the study region

Fig.4 Magnitude versus time for earthquakes $(M \geq 2.0)$ used in the analysis

Fig. 5 The length of day relating to the Earth's rotation versus time. (a) The raw data (coming from EOC). (b) The seasonal variations in the length of day relating to the Earth's rotation (filtered with period range from $120 \mathrm{~d}$ to $370 \mathrm{~d}$ )

Fig. 6 Calculation of the phase angle." + "indicates the occurring time of an earthquake

Fig. $7 \quad P$-value as a function of time for earthquakes $(2.0 \leq M \leq 2.4)$ that occurred in the study region (the downward arrow ' $\downarrow$ ' shows the occurrence time of the $M 7.1$ Ridgecrest event.The red area indicates the $95 \%$ confidence range as resulting from bootstrap analysis). (b) Histogram showing the frequency of the phase angle of earthquakes occurring six years before the $M 7.1$ Ridgecrest earthquake. Thick solid curve denotes sinusoidal function fitted to the frequency distribution. 
Fig. $8 P$-value as a function of time for earthquakes of $M \geq 2.5$ that occurred in the study region. The downward arrow ' $\downarrow$ ' shows the occurrence time of the $M 7.1$ Ridgecrest event. The red area indicates the $95 \%$ confidence range as resulting from bootstrap analysis.

393

394

395

396

397

398

399

400

401

402

403

404

405

406

407

408

409

410

411

412

413

Fig. 9 Spacial distribution of $P$-values in the six years prior to the $M 7.1$ Ridgecrest earthquake (A spacial window of $50 \mathrm{~km} \times 50 \mathrm{~km}$ moving by $2 \mathrm{~km}$ in both along-latitude and along-longitude directions. The star shows the epicenter of the $M \mathrm{w} 7.1$ Ridgecrest earthquake). Solid lines show the rupture zone,and dashed lines the study region. (a) $P>0$. (b) $P<0.5 \%$.

Fig. $10 R$ as a function of time for earthquakes $(2.0 \leq M \leq 2.4)$ that occurred in the study region. The downward arrow ' $\downarrow$ ' shows the occurrence time of the $M 7.1$ Ridgecrest event. The light green area indicates the $95 \%$ confidence range between $R_{\mathrm{L}}$ and $R_{\mathrm{U}}$.

Fig.11 Map showing the seasonal variations of LOD (length of day) and $a_{e}$ (the linear acceleration at the equator). The solid curve denotes LOD and the dashed one $a_{e}$. Two boxes show the phase angle range between $-50^{\circ}$ and $130^{\circ}$. (1)

(1)
(1) 
Figures
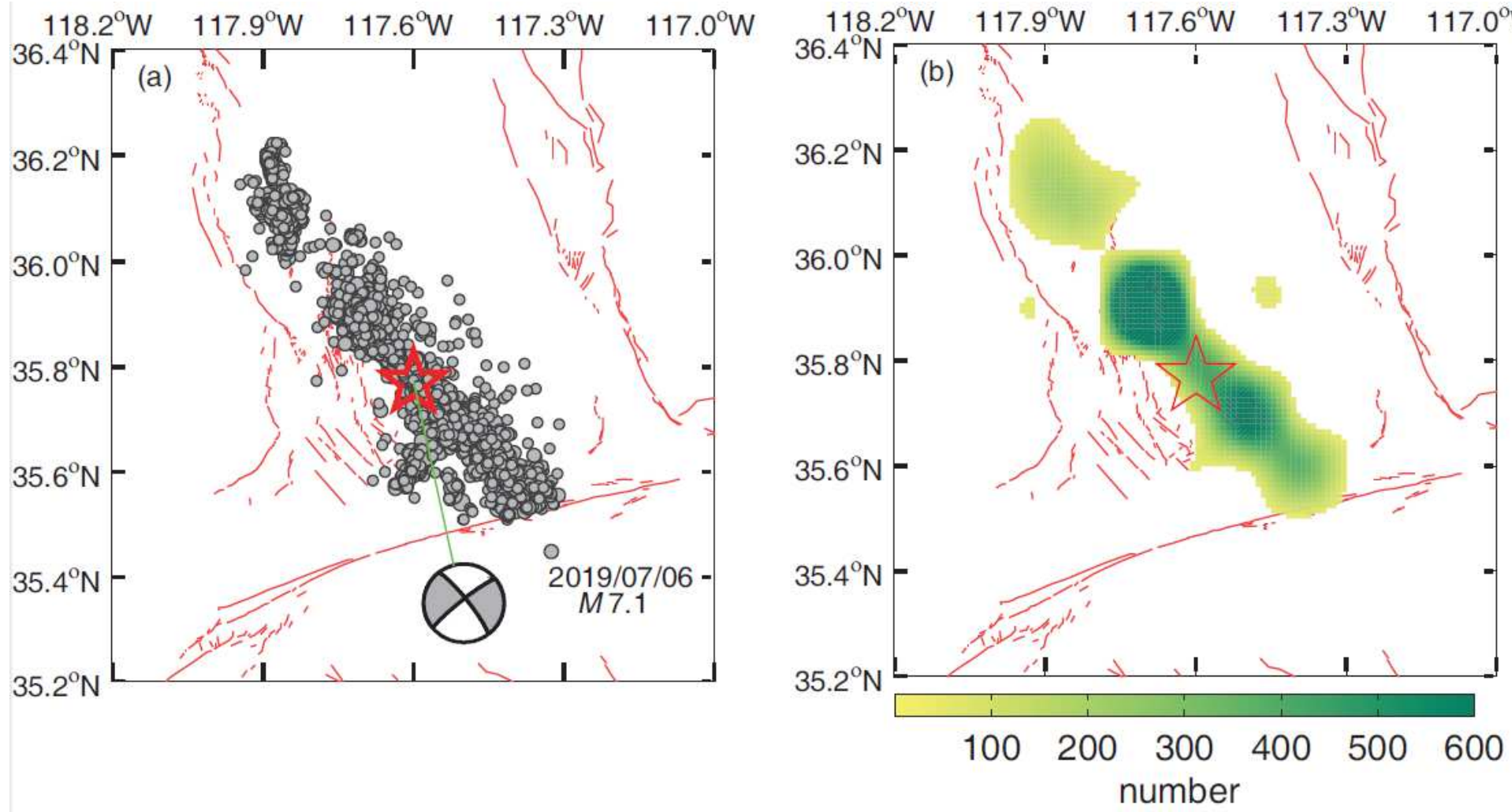

Figure 1

Maps showing the locations of aftershocks and their spacial number distribution,regional faults are shown as red lines,the red star shows the epicenter of the M 7.1 Ridgecrest earthquake,the focal mechanism solution comes from the Global Centroid Moment Tensor Project [www.globalcmt.org/CMTsearch.html]. (a) The locations of aftershocks $(M \geq 2.0)$ that occurred from 6 July to 31 August following the M 7.1 Ridgecrest earthquake. (b) The spacial number distribution for earthquakes in Fig. $1 \mathrm{a}$, a spatial window of $0.1^{\circ} \times 0.1^{\circ}$ is moved by $0.01^{\circ}$ in both the along-latitude and along-longitude directions. 


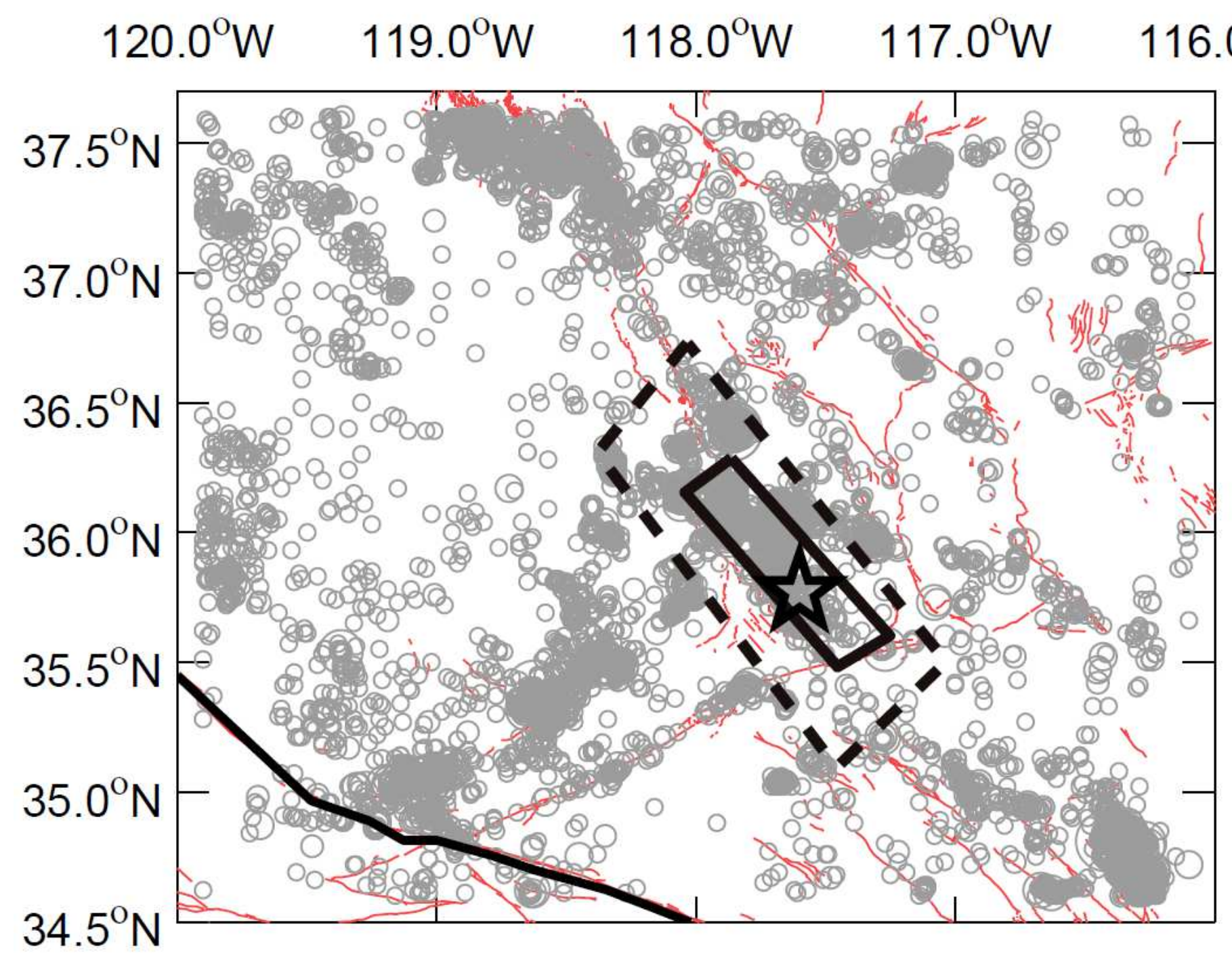

Figure 2

Map showing location of the study region ( surrounded by dashed lines) and its tectonic setting.Regional faults are shown as red lines. The bold black line located at the lower left shows the plate boundary. Black circles show the epicenters of earthquakes (ML $\geq 2.0$ ) that occurred from January 2000 to Jun 2019. The star represents the epicenter of the M 7.1 Ridgecrest mainshock and solid black lines show the rupture zone. 


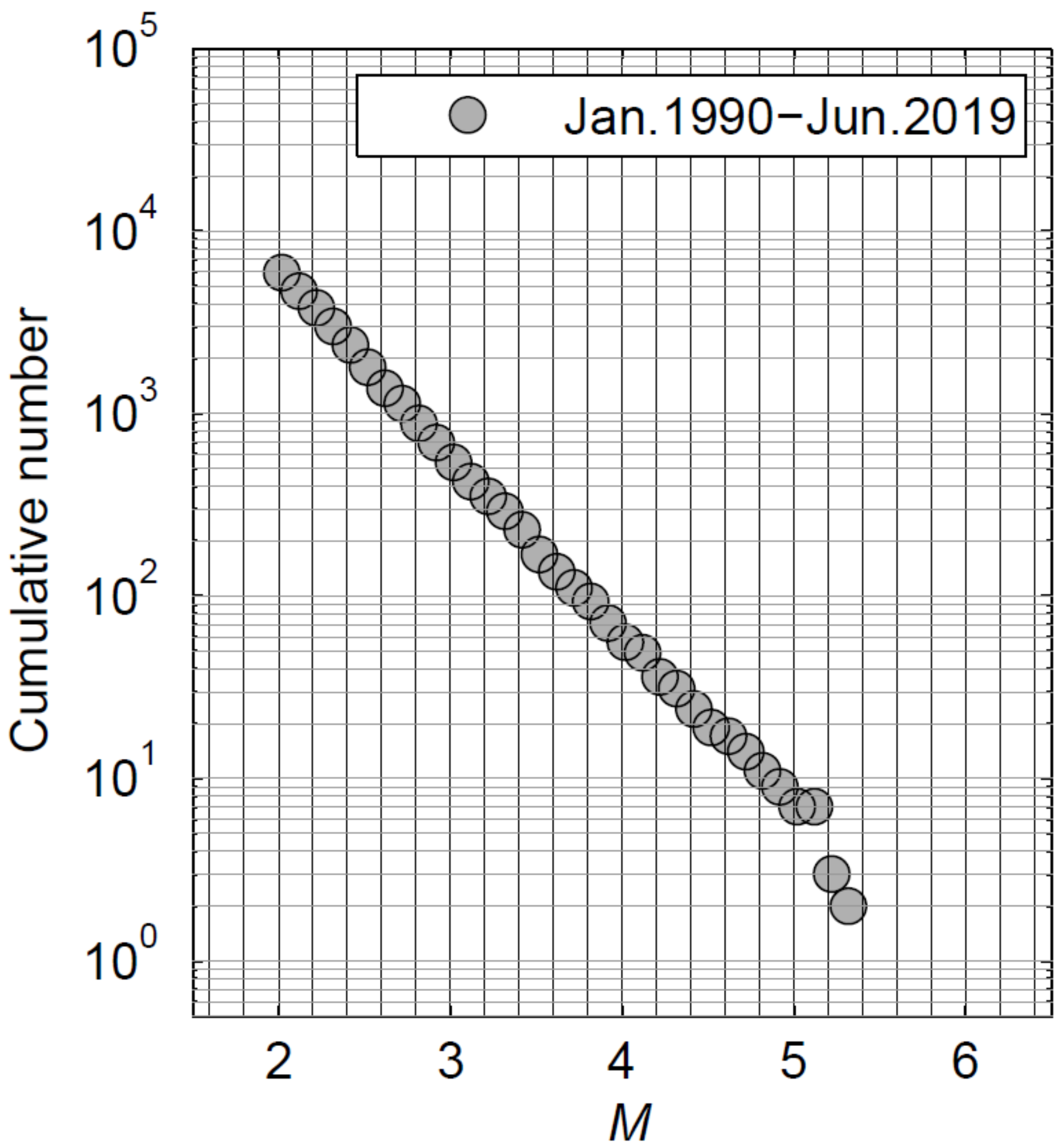

Figure 3

Cumulative number versus magnitude for earthquakes in the study region 


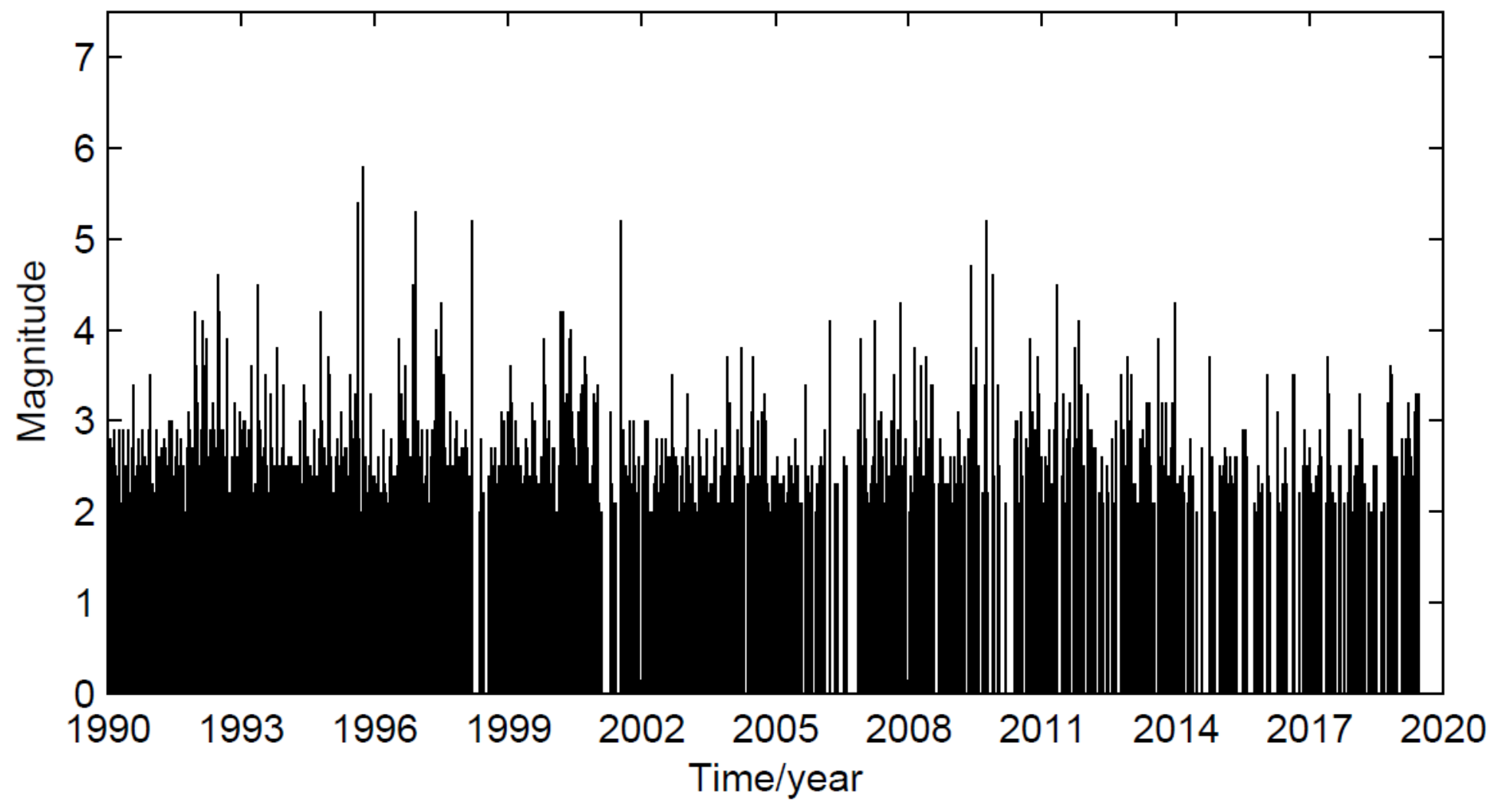

Figure 4

Magnitude versus time for earthquakes $(M \geq 2.0)$ used in the analysis 

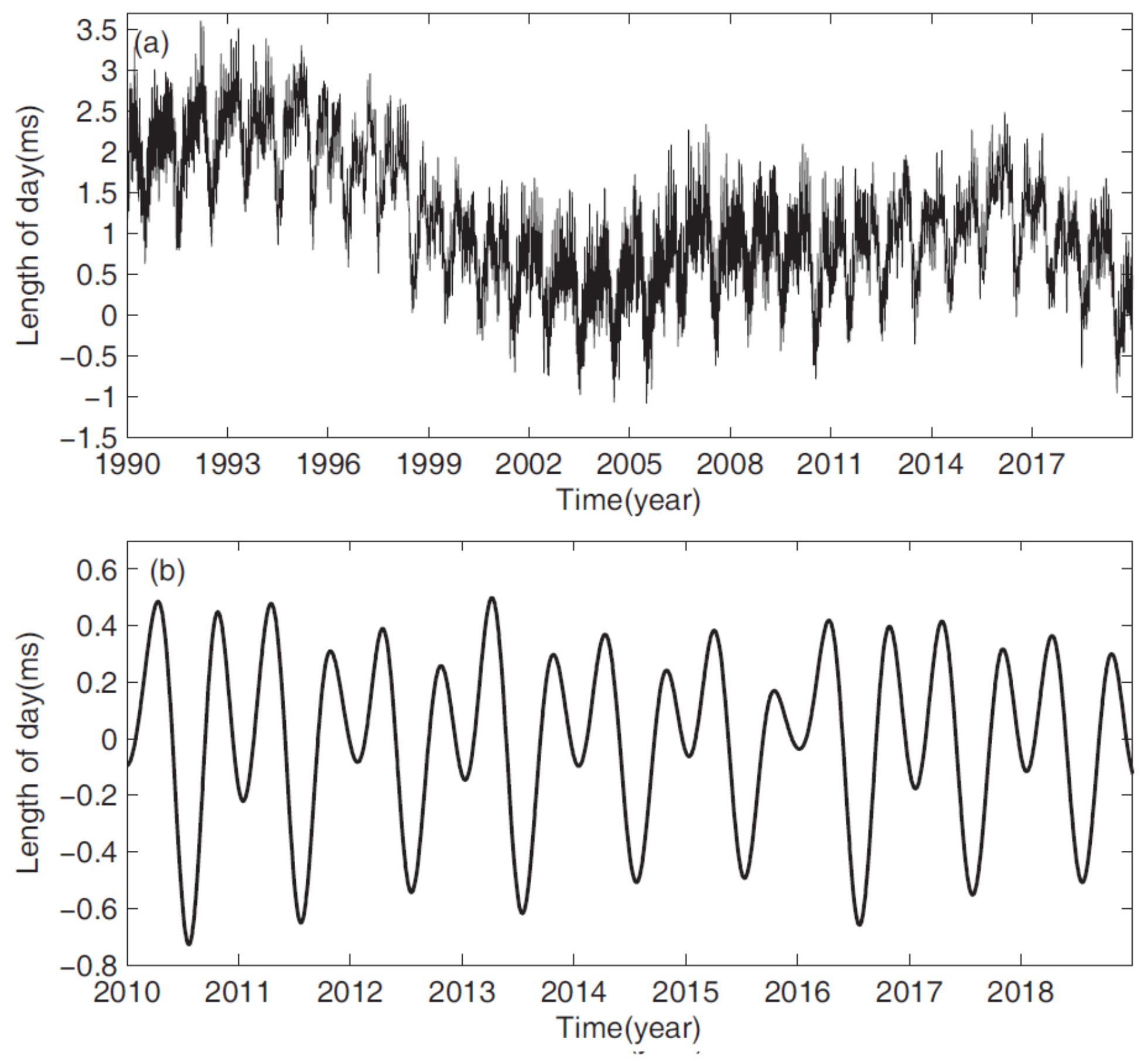

Figure 5

The length of day relating to the Earth's rotation versus time. (a) The raw data (coming from EOC). (b) The seasonal variations in the length of day relating to the Earth's rotation (filtered with period range from $120 d$ to $370 d$ ) 


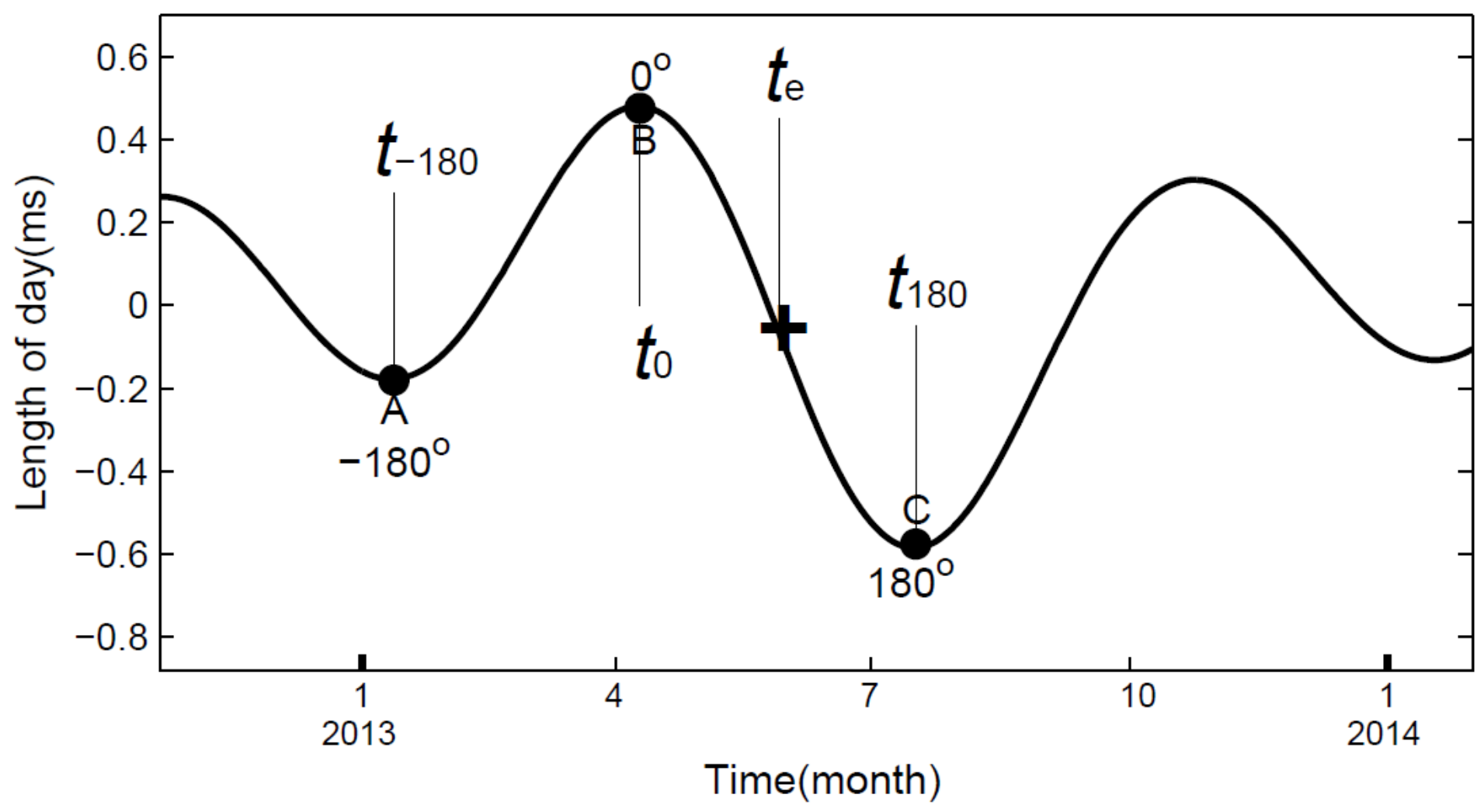

Figure 6

Calculation of the phase angle." +"indicates the occurring time of an earthquake
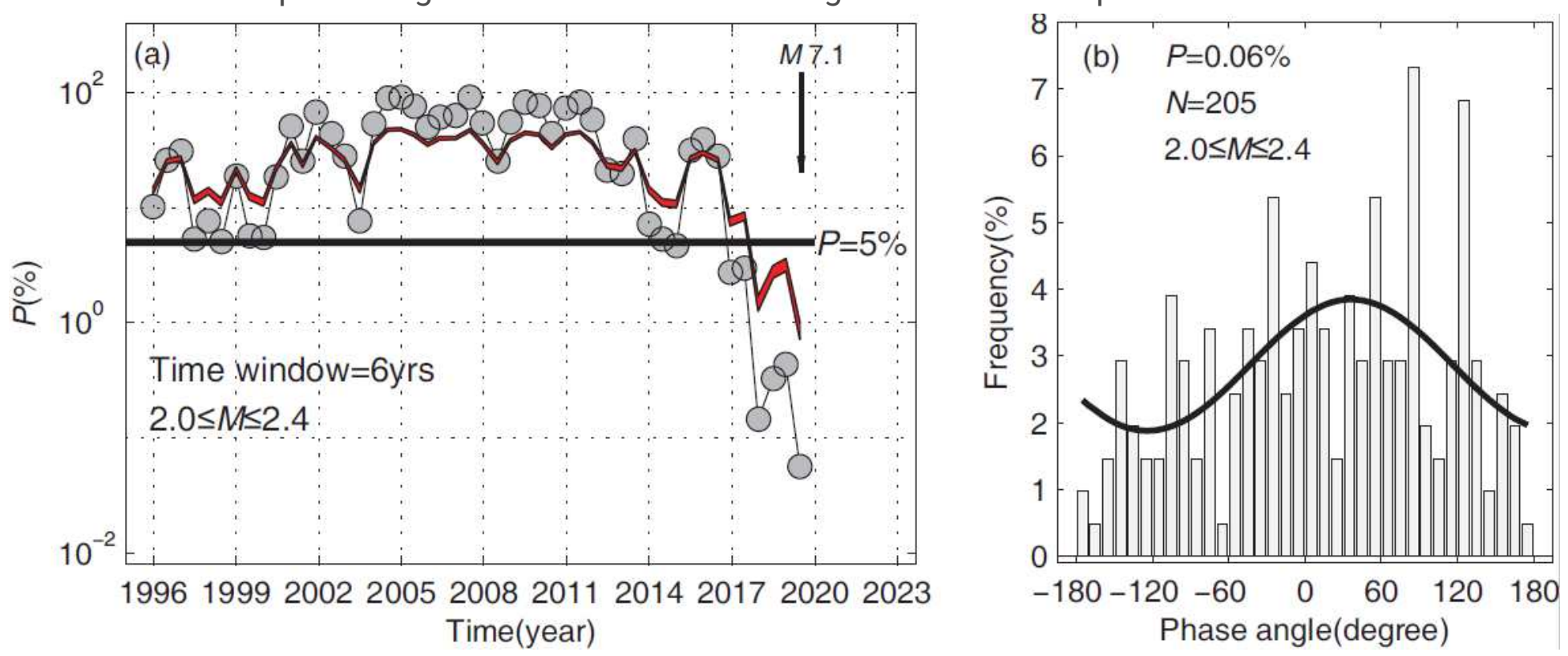

Figure 7

P-value as a function of time for earthquakes $(2.0 \leq \mathrm{M} \leq 2.4)$ that occurred in the study region (the downward arrow ' $\square$ ' shows the occurrence time of the M 7.1 Ridgecrest event.The red area indicates the $95 \%$ confidence range as resulting from bootstrap analysis). (b) Histogram showing the frequency of the 
phase angle of earthquakes occurring six years before the M 7.1 Ridgecrest earthquake. Thick solid curve denotes sinusoidal function fitted to the frequency distribution.

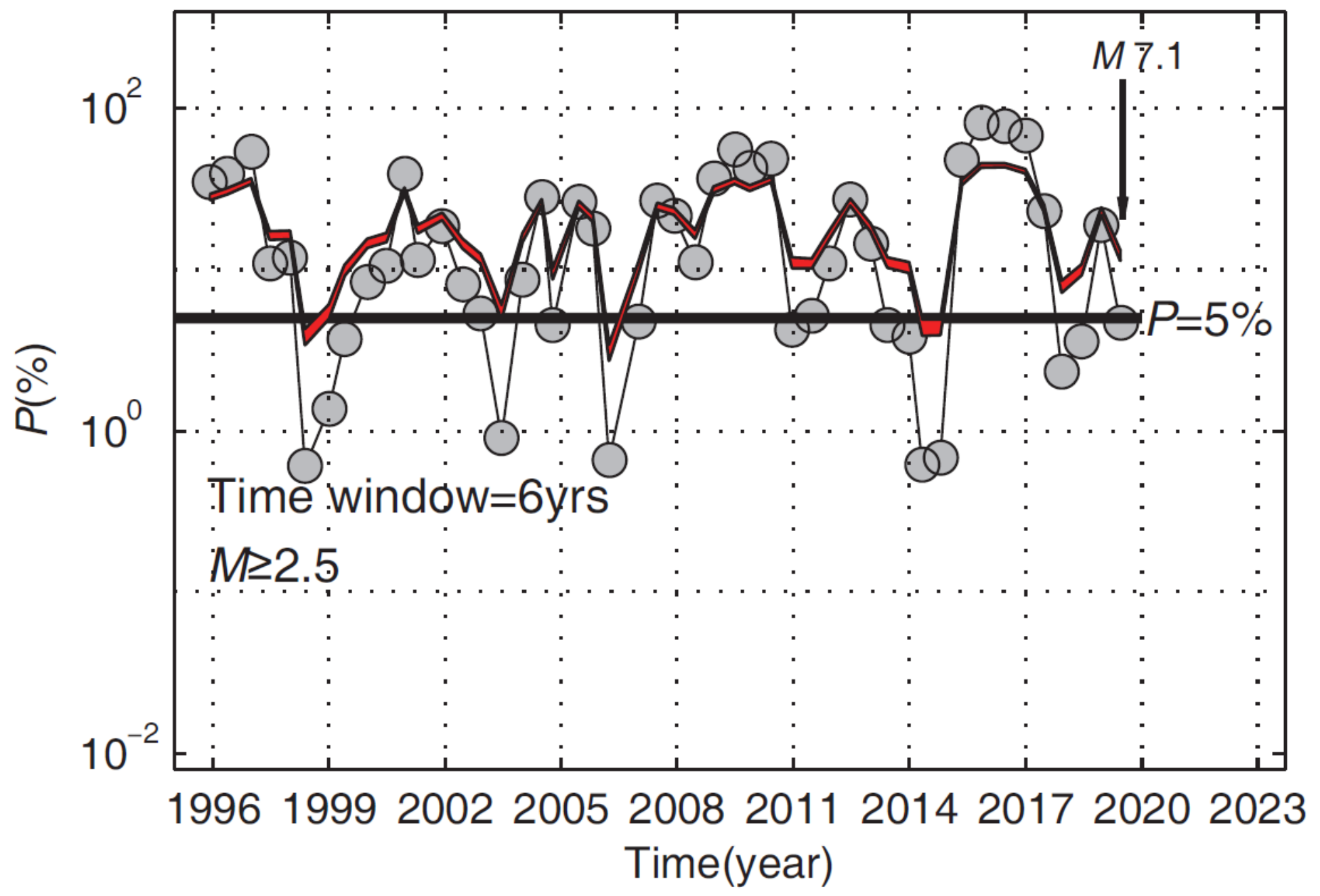

Figure 8

P-value as a function of time for earthquakes of $\mathrm{M} \geq 2.5$ that occurred in the study region. The downward arrow ' $₫$ ' shows the occurrence time of the M 7.1 Ridgecrest event. The red area indicates the $95 \%$ confidence range as resulting from bootstrap analysis. 

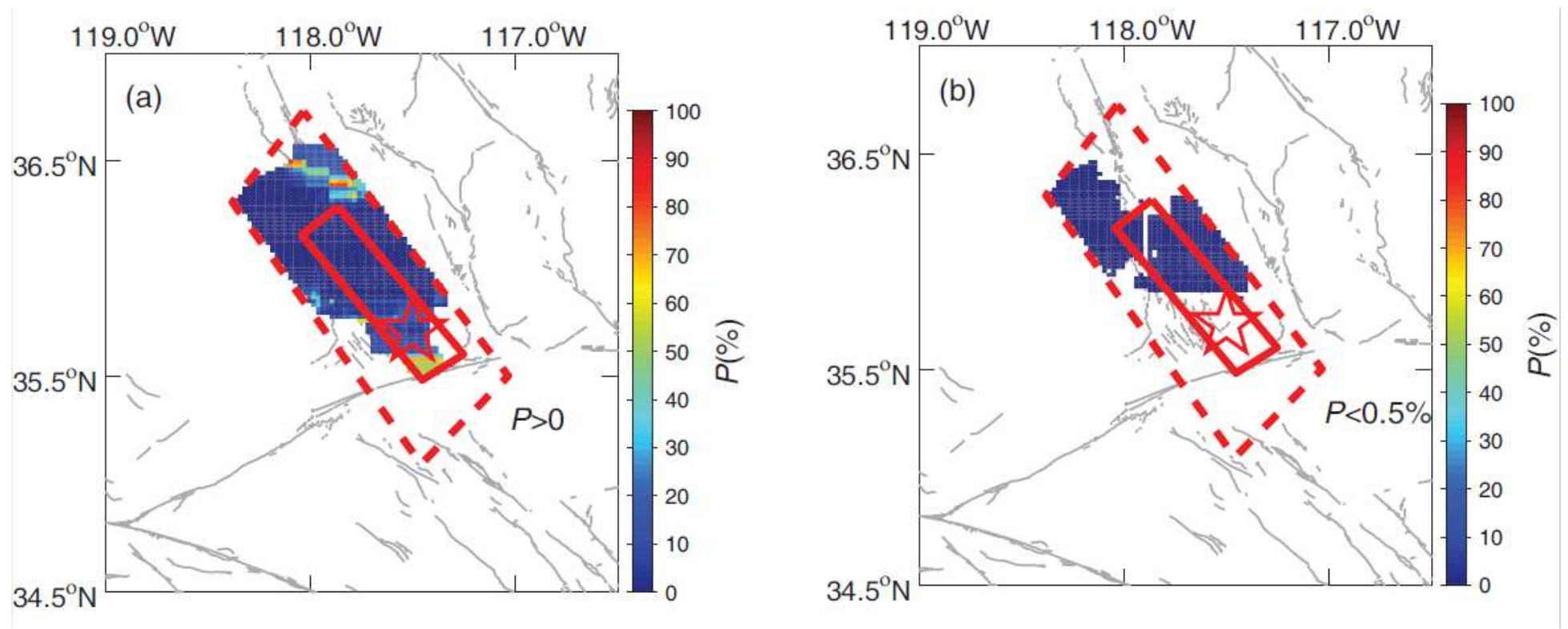

Figure 9

Spacial distribution of P-values in the six years prior to the M 7.1 Ridgecrest earthquake (A spacial window of $50 \mathrm{~km} \times 50 \mathrm{~km}$ moving by $2 \mathrm{~km}$ in both along-latitude and along-longitude directions. The star shows the epicenter of the Mw 7.1 Ridgecrest earthquake). Solid lines show the rupture zone,and dashed lines the study region. (a) $P>0$. (b) $P<0.5 \%$. 


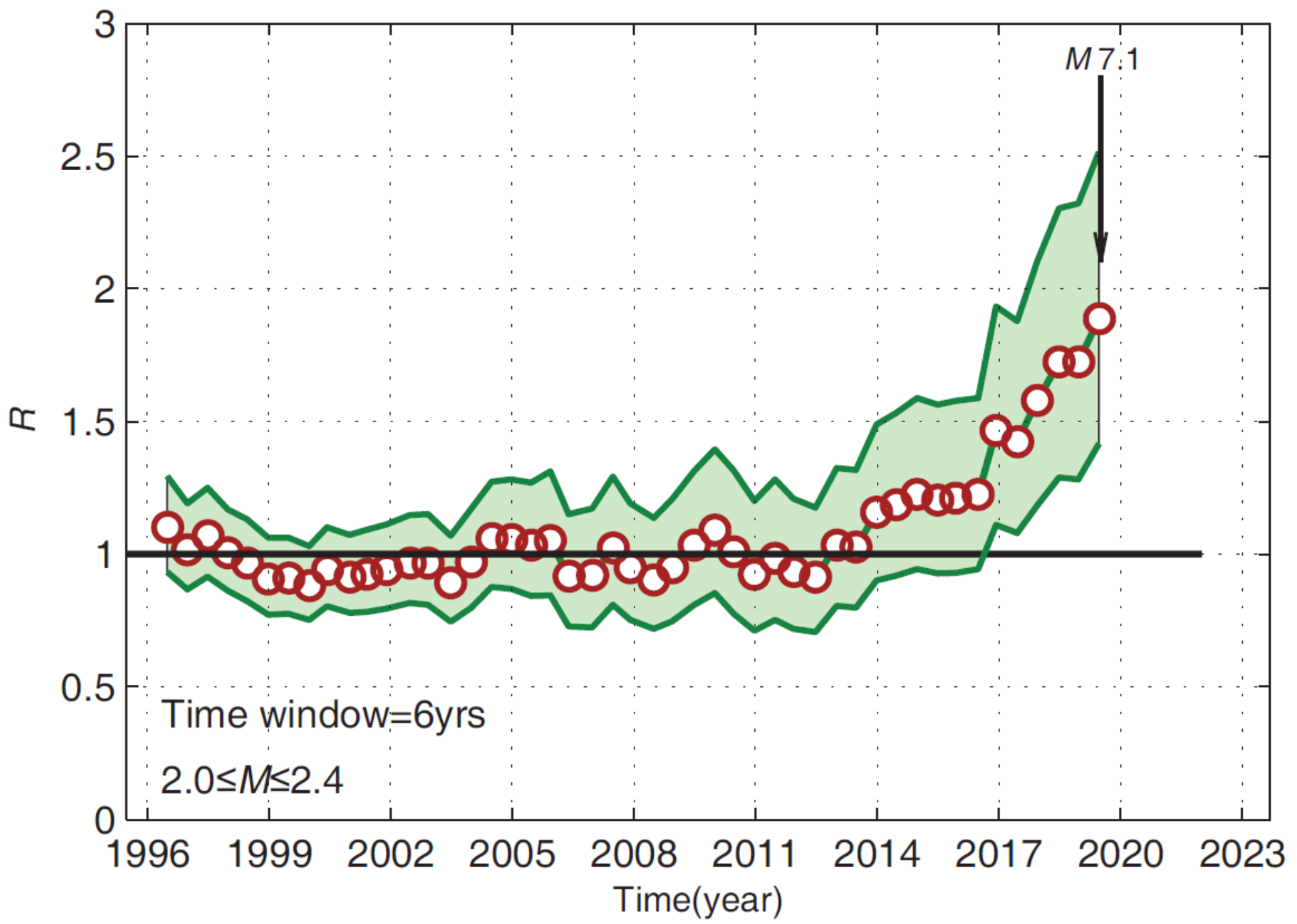

Figure 10

$R$ as a function of time for earthquakes $(2.0 \leq M \leq 2.4)$ that occurred in the study region. The downward arrow ' $\$ ' shows the occurrence time of the M 7.1 Ridgecrest event. The light green area indicates the $95 \%$ confidence range between $\mathrm{RL}$ and $\mathrm{RU}$. 


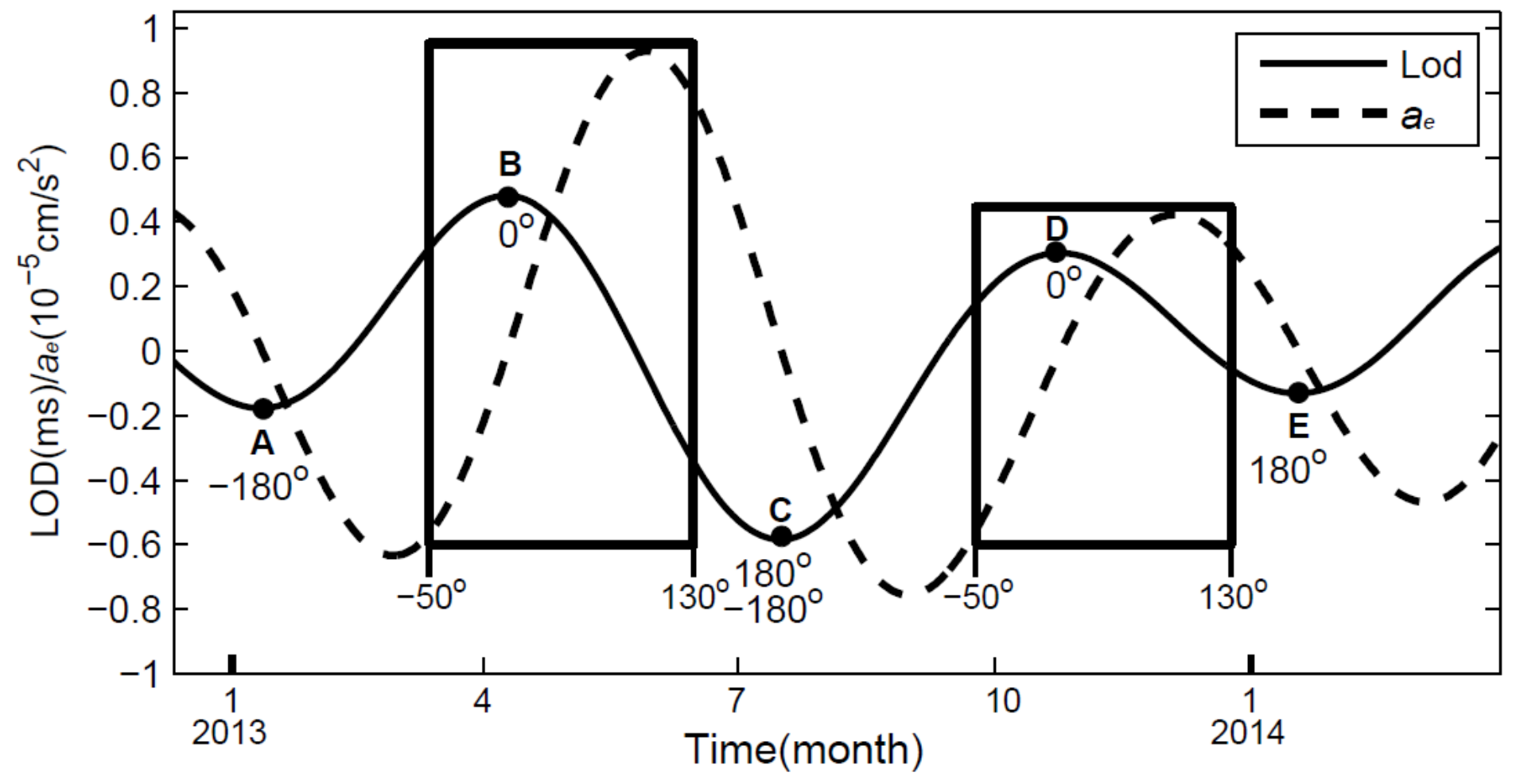

Figure 11

Map showing the seasonal variations of LOD (length of day) and ae (the linear acceleration at the equator). The solid curve denotes LOD and the dashed one ae.Two boxes show the phase angle range between $-50^{\circ}$ and $130^{\circ}$. 